\title{
ON THE CONTINUOUS MONOTONIC DECOMPOSITION OF SOME COMPLETE TRIPARTITE GRAPHS
}

\author{
Joseph Varghese, A. Antonysamy
}

\begin{abstract}
Let $G=(v, \mathcal{E})$ be a connected simple graph of order $p$ and size $q$. If $H_{1}, H_{2}, \ldots, H_{k^{\prime}} k \in \mathbb{N}$ are edge-disjoint subgraphs of $G \ni \mathcal{E}(G)=\mathcal{E}\left(H_{1}\right) \cup \mathcal{E}\left(H_{2}\right) \cup \ldots . \cup \mathcal{E}\left(H_{k}\right)$, then $H_{1}, H_{2}, \ldots . H_{k}$ is said to be a decomposition of $G$. Ascending Subgraph Decomposition (ASD) is a decomposition of $G$ into subgraph $H_{i}$ (not necessarily connected) $\exists \mathcal{E}\left(\mathrm{H}_{i}\right) \mid=i$ and is isomorphic to a proper subgraph of $H_{i+1} . A$ decomposition, $\left\{H_{1} H_{2}, \ldots . . H_{k}, \forall k \in N\right\}$, is said to be a Continuous Monotonic Decomposition (CMD) if each $H_{i}$ is connected and $\mathcal{E}\left(H_{i}\right)=i$ for each $i \in \mathbb{N}$. Necessary and Sufficient Conditions for $K_{1,3, m^{\prime}}$ $K_{2,3 m^{\prime}} K_{2,5 m}$ and $K_{3,5, m}$ to accept $C M D$.
\end{abstract}

Key words: Graph Theory, Graph Decomposition, Complete Tripartite Graph, Continuous Monotonic Decomposition, Triangular Numbers.

* Research Scholar, St. Xavier's College, Manonmaniam Sunudarnar University, Tirunelveli; (email: josephvk@gmail.com).

- Research Department of Mathematics, St Xavier's College, Palayamkotai, India (e-mail: fr_antonysamy@hotmail.com). 


\section{Introduction}

An undirected graph with the property that there is a path between every pair of vertices is known as a connected graph. A graph $G$, referred to here is an undirected connected graph without loops or multiple edges. The degree of a vertex $u$ of any graph is the number of edges incident with $u$ and is denoted by $d(u)$ and the distance between two vertices $u$ and $v$ of $G$ is the length of the shortest $u-v$ path in $G$ and is denoted by $d(u, v)$. A graph $G$ is called n-regular graph if $\operatorname{deg}(v)=n \in \mathbb{N}, \forall v \in V(G)$.

A complete graph with vertices $n \in \mathbb{N}$, denoted by $K_{n}$, is a connected simple graph with every vertex is connected with every other vertex by an edge. A graph with $n$ vertices $v_{1}, v_{2}, \ldots v_{n^{\prime}}$, where $n \geq 3$, and edges $\left\{v_{1}, v_{2}\right\},\left\{v_{2}, v_{3}\right\}, \ldots,\left\{v_{n-1}, v_{n}\right\},\left\{v_{n^{\prime}}\right.$ $\left.v_{1}\right\}$ is known as a cycle, $C_{n}$.

A path of length $t$ is denoted by $P_{t}$. A complete $m$-partite graph $G=K_{n 1, n 2, \ldots . . n m}$ $\forall n_{1}, n_{2}, \ldots n_{m} \in \mathbb{N}$ is a graph whose vertex set $V$ can be partitioned into $m$ subsets $V_{1}, V_{2}, \ldots V_{m}$ such that every edge of $G$ joins every vertex of $V_{i}$ with every vertex of $V_{j}$ where $i \neq i$ and $\left|V_{i}\right|=i$. When $m=2, G$ is a complete bipartite graph and $m=3$, $G$ is a complete tripartite graph. Terms not defined here are used in the sense of Harary [1].

\section{Graph Decompositions}

Let $G=(V, \mathcal{E})$ be a connected simple graph of order $p$ and size $q$. If $H_{1}, H_{2}, \ldots H_{k}$ $\forall k \in \mathbb{N}$ are edge-disjoint subgraphs of $G \ni \mathcal{E}(G)=\mathcal{E}\left(\mathrm{H}_{1}\right) \dot{U} \mathcal{E}\left(\mathrm{H}_{2}\right)$, $\cup \ldots . . \cup \mathcal{E}\left(H_{k}\right)$, then $H_{1}, H_{2}, \ldots . . H_{k}$ is said to be a decomposition of $G$. Different types of decomposition of $G$ have been studied in the literature by imposing suitable conditions on the subgraphs $\mathrm{H}_{\text {; }}$.

Alavi et al [2], introduced Ascending Subgraph Decomposition (ASD) as a decomposition of $G$ into subgraph $H_{i}$ (not necessarily connected) $\exists\left|\mathcal{E}\left(H_{i}\right)\right|=i$ and is isomorphic to a proper subgraph of $\mathrm{H}_{i+1}$. Gnana Dhas and Paulraj Joseph introduced a new concept known as continuous monotonic decomposition of graphs [3]. A decomposition, $\left\{H_{1}, H_{2}, \ldots . H_{k}\right\} \forall k \in \mathbb{N}$, is said to be a Continuous Monotonic Decomposition (CMD) if each $H_{i}$ is connected and 
$\left|\mathcal{E}\left(H_{i}\right)\right|=i \forall i \in \mathbb{N}$. If $G$ admits a $C M D,\left\{H_{3}, H_{4}, \ldots . . H_{k}\right\} \forall k \in \mathbb{N}$, where each $H_{i}$ is a cycle of length $i$ in $G$, then we say that $G$ admits Continuous Monotonic Cycle Decomposition (CMCD) [4]. A CMD in which each $\mathrm{H}_{i}$ is a star is said to be a Continuous Monotonic Star Decomposition (CMSD) and a CMD in which each $H_{i}$ is a path is said to be a Continuous Monotonic Path Decomposition (CMPD) [3].

\section{Example 2.1}
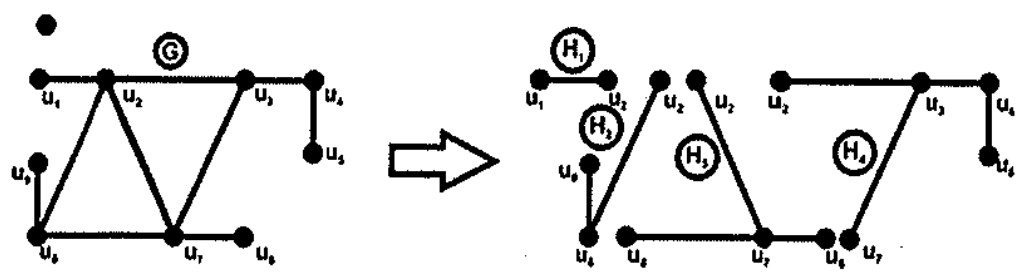

Fig. 2.1 Continuous Monotonic Decomposition of $\mathrm{G}$ into $\mathrm{H}_{1}, \mathrm{H}_{2}, \mathrm{H}_{3}$, and $\mathrm{H}_{4}$

\section{Triangular Numbers}

Triangular number is a natural number that is the sum consecutive natural numbers, beginning with 1. Pythagoras found that number is triangular if and only if it is of the form $\frac{n(n+1)}{2}$ for some $n \geq 1$. Plutarch stated that $n$ is a triangular number if and only if $8 n+1$ is a perfect square. The square of any integer is either of the form $3 k$ or $3 k+1$ for some $k \in \mathbb{N}$.

Euler identified that if $n$ is a triangular number, then so are $9 n+1,25 n+3$ and $49 n+6$. If $t_{n}$ denotes the $n^{\text {th }}$ triangular number, then $t_{n}=\left({ }^{(n+1)} C_{2}\right.$. All these number theory results are used in the sense of David M. Burton [6].

\section{Continuous Monotonic Decomposition of Some Complete Tripartite Graphs}

Continuous Monotonic Decomposition of a wide variety of graphs had been studied by Gnana Dhas and Paulraj Joseph, and Navaneetha Krishnan and Nagarajan [3]-[5]. If a graph $\mathrm{G}$ admits a CMD $\left\{\mathrm{H}_{1}, \mathrm{H}_{2}, \ldots \ldots \mathrm{H}_{\mathrm{k}}\right\} \forall \mathrm{k} \in \mathbb{N}$ if and only if $q==^{(n+1)} \mathrm{C}_{2}[3]$. But we know that for any positive integer $n,(n+1) C_{2}$ is a triangular number. Hence, 
if we are able to find out the number of the edges of any connected graph, it is easy for us to conclude whether it admits CMD or not. In this paper, I am presenting the necessary and sufficient condition for a collection of complete tripartite graphs and tensor product of Graphs which admit CMD.

The following four results are about particular classes of complete tripartite graphs which accept $C M D$.

Theorem 4.1 A complete tripartite graph $\mathrm{K}_{1,3, \mathrm{~m}}$ accepts $\mathrm{CMD}$ of $\mathrm{H}_{1}, \mathrm{H}_{2}, \ldots$, $H_{4 n+1}$ if and only if $m=\left(4 n^{2}+3 n-1\right) / 2$ when $n$ is odd and $C M D$ of $H_{1}, H_{2}, \ldots \ldots$, $H_{4 n+2}$ if and only if $m=\left(4 n^{2}+5 n\right) / 2$ when $n$ is even $\forall n \in \mathbb{N}$.

Proof. Assume that a complete tripartite graph $K_{1,3, m}$ accepts CMD of $H_{1}, H_{2}$, $\ldots . ., H_{4 n+1}$ when $n$ is odd and $C M D$ of $H_{1}, H_{2}, \ldots, H_{4 n+2}$ when $n$ is even, $\forall n \in \mathbb{N}$.

We have, $q\left(K_{1,3, m}\right) \quad=[m(1+3)+1(m+3)+3(m+1)] / 2$

$$
=4 \mathrm{~m}+3 \forall \mathrm{m} \in \mathbb{N} \text {...... }
$$

We know that $G$ accepts $C M D H_{1}, H_{2}, \ldots ., H_{n}$ iff $q(G)=n(n+1) / 2, \forall n \in \mathbb{N}$.

Case 1: when $\mathrm{n}$ is odd

$K_{1,3, m}$ accepts $C M D H_{1}, H_{2}, \ldots ., H_{4 n+1}$ iff $q\left(K_{1,3, m}\right)=(4 n+1)(4 n+2) / 2$ where $n \in \mathbb{N}$ and $n$ odd.

i.e., $=(4 n+1)(2 n+1)$, for $n \in \mathbb{N} \ldots .$. and $n$ odd

i.e., $q\left(K_{1,3, \mathrm{~m}}\right)$ must be a member of the sequence $1,3,6,10$,

$15, \ldots k(k+1) / 2 \forall k \in \mathbb{N}$.

i.e., $(4 n+1)(2 n+1)=k(k+1) / 2$ for some $k \in \mathbb{N}$ and $n \in \mathbb{N}$ and $n$ odd.

i.e., $k=4 n+1$ for $n \in \mathbb{N}$ and $n$ odd....

Also, $\quad K_{1,3, \mathrm{~m}}$ accepts $C M D$ iff $q\left(K_{1,3, m}\right)$ is one among the members of the sequence (3).

i.e., $4 m+3$ should be one of these values.... using (1) and (3) 


$$
\begin{aligned}
& \text { i.e., } 4 m+3=k(k+1) / 2 \text { for some } k \in \mathbb{N} \text {. } \\
& \text { i.e., } 4 m+3=(4 n+1)(2 n+1) \ldots \text { using }(4) \\
& \text { i.e., } 4 m=(4 n+1)(2 n+1)-3 \text {, for } n \in \mathbb{N} \text { and } n \text { odd. } \\
& \text { i.e., } m=\left(4 n^{2}+3 n-1\right) / 2 \text { for } n \in \mathbb{N} \text { and } n \text { odd. }
\end{aligned}
$$

The values of $m$ are $3,22,57,108,175 \ldots \ldots$

\section{Example 4.1}

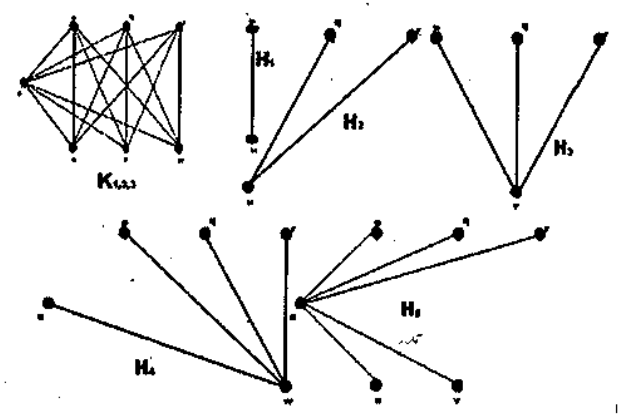

Fig 4.1: Continuous Monotonic Decomposition of $\mathrm{K}_{1,3,3}$

Case 2: when $n$ is even

$\mathrm{K}_{1,3, \mathrm{~m}}$ accepts $\mathrm{CMD} \mathrm{H} \mathrm{H}_{1} \mathrm{H}_{2}, \ldots \ldots, \mathrm{H}_{4 n+2}$ iff $\mathrm{q}\left(\mathrm{K}_{1,3, \mathrm{~m}}\right)=(4 n+3)(4 n+2) / 2$ where $n$ $\in \mathbb{N}$ and $n$ even.

$$
\text { i.e., }=(4 n+3)(2 n+1), \text { for } n \in \mathbb{N} \text { and } n \text { even..... }
$$

i.e., $q\left(K_{1,3, m}\right)$ must be a member of the sequence $1,3,6,10,15, \ldots k(k+1) /$ $2 \forall k \in \mathbb{N}$.

i.e., $(4 n+3)(2 n+1)=k(k+1) / 2$ for some $k \in \mathbb{N}$ and $n \in \mathbb{N}$ and $n$ even.

i.e., $k=4 n+2$ for $n \in \mathbb{N}$ and $n$ even.... 
Also, $\quad K_{1,3, m}$ accepts $C M D$ iff $q\left(K_{1,3, m}\right)$ is one among the members of the sequence (3).

i.e., $4 m+3$ should be one of these values..... using (1) and (3)

i.e., $4 m+3=k(k+1) / 2$ for some $k \in \mathbb{N}$.

i.e., $4 m+3=(4 n+3)(2 n+1) \ldots$. using $(4)$

i.e., $4 m=(4 n+3)(2 n+1)-3$, for $n \in \mathbb{N}$ and $n$ even.

i.e., $m=\left(4 n^{2}+5 n\right) / 2$ for $n \in \mathbb{N}$ and $n$ even.

The values of $m$ are $13,42,87,148,225 \ldots \ldots$.

\section{Example 4.2}

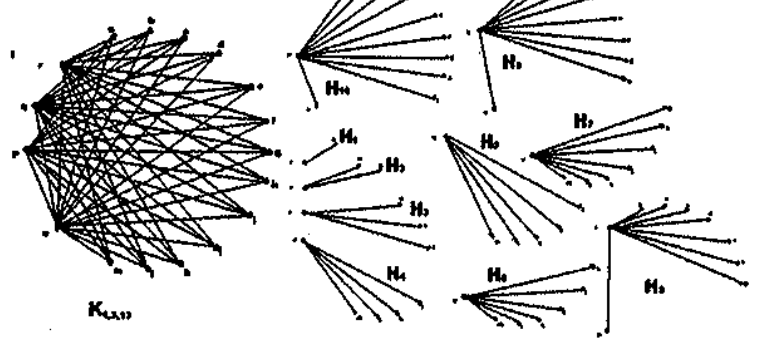

Fig 4.2: Continuous Monotonic Decomposition of $K_{1,3,13}$

Hence, a complete tripartite graph $K_{1,3, m}$ accepts $C M D$ of $H_{1}, H_{2}, \ldots, H_{4 n+1}$ if $m=\left(4 n^{2}+3 n-1\right) / 2$ when $n$ is odd and $C M D$ of $H_{1}, H_{2}, \ldots, H_{4 n+2}$, if $m=\left(4 n^{2}+5 n\right) /$ 2 when $n$ is even, $\forall n \in \mathbb{N}$

Conversely,

Suppose that $K_{1,3, m}$ is a complete tripartite graph with $m=\left(4 n^{2}+3 n-1\right) / 2$ when $n$ is odd and $m=\left(4 n^{2}+5 n\right) / 2$ when $n$ is even, $\forall n \in \mathbb{N}$

We know that $q\left(K_{1,3, m}\right)=4 m+3$ 
Case 1: When $m=\left(4 n^{2}+3 n-1\right) / 2$

$$
\begin{aligned}
q\left(K_{1,3, m}\right) & =4 m+3 \\
& =4\left(4 n^{2}+3 n-1\right) / 2+3 \\
& =\left(8 n^{2}+6 n-2\right)+3 \\
& =\left(8 n^{2}+6 n+1\right) \\
& =2 n(4 n+1)+(4 n+1) \\
& =(2 n+1)(4 n+1) \ldots \ldots . .
\end{aligned}
$$

(4) is of the form $k(k+1) / 2 \forall k \in \mathbb{N}$.

This implies that $K_{1,3, m}$ being a connected simple graph, can be decomposed into $H_{1}, H_{2}, \ldots . . H_{k} \forall k \in \mathbb{N}$.

i.e., $K_{1,3, m}$ can be decomposed into $H_{1}, H_{2}, \ldots . H_{4 n+1}$ for $n \in \mathbb{N}$ and $n$ odd.

Case 2: When $m=\left(4 n^{2}+5 n\right) / 2$

$$
\begin{aligned}
q\left(K_{1,3, m}\right) & =4 m+3 \\
& =4\left(4 n^{2}+5 n\right) / 2+3 \\
& =\left(8 n^{2}+10 n\right)+3 \\
& =2 n(4 n+3)+4 n+3 \\
& =(2 n+1)(4 n+3) \ldots \ldots . .
\end{aligned}
$$

(5) is of the form $k(k+1) / 2 \forall k \in \mathbb{N}$.

This implies that $K_{1,3, \mathrm{~m}}$ being a connected simple graph, can be decomposed into $H_{1}, H_{2}, \ldots . . H_{k} \forall k \in \mathbb{N}$.

i.e., $K_{1,3, m}$ can be decomposed into $H_{1}, H_{2}, \ldots . H_{4 n+2,}$ for $n \in \mathbb{N}$ and $n$ even. 
Table 4.1 First $25 \mathrm{~K}_{1,3, \mathrm{~m}}$ 's which admit CMD and their CMDs

\begin{tabular}{|c|c|c|}
\hline $\mathrm{m}$ & $q\left(K_{1,3, m}\right)$ & $C M D$ \\
\hline 3 & 15 & $H_{1}, H_{2}, \ldots . H_{5}$ \\
\hline 13 & 55 & $\mathrm{H}_{1}, \mathrm{H}_{2}, \ldots . \mathrm{H}_{10}$ \\
\hline 22 & 91 & $H_{1}, H_{2}, \ldots . H_{13}$ \\
\hline 42 & 171 & $\mathrm{H}_{1}, \mathrm{H}_{2}, \ldots . \mathrm{H}_{18}$ \\
\hline 57 & 231 & $\mathrm{H}_{1}, \mathrm{H}_{2^{\prime}} \ldots \ldots / \mathrm{H}_{21}$ \\
\hline 87 & 351 & $\mathrm{H}_{1}, \mathrm{H}_{2}, \ldots . \mathrm{H}_{26}$ \\
\hline 108 & 435 & $\mathrm{H}_{1}, \mathrm{H}_{2}, \ldots . \mathrm{H}_{29}$ \\
\hline 148 & 595 & $H_{1}, H_{2}, \ldots . H_{34}$ \\
\hline 175 & 703 & $\mathrm{H}_{1}, \mathrm{H}_{2}, \ldots . \mathrm{H}_{37}$ \\
\hline 225 & 903 & $\mathrm{H}_{1}, \mathrm{H}_{2}, \ldots . \mathrm{H}_{42}$ \\
\hline 258 & 1035 & $H_{1}, H_{2}, \ldots . H_{45}$ \\
\hline 318 & 1275 & $\mathrm{H}_{1}, \mathrm{H}_{2}, \ldots . \mathrm{H}_{50}$ \\
\hline 357 & 1431 & $H_{1}, H_{2}, \ldots . H_{53}$ \\
\hline 427 & 1711 & $H_{1}, H_{2}, \ldots . H_{58}$ \\
\hline 472 & 1891 & $\mathrm{H}_{1}, \mathrm{H}_{2}, \ldots . \mathrm{H}_{61}$ \\
\hline 552 & 2211 & $\mathrm{H}_{1}, \mathrm{H}_{2}, \ldots . \mathrm{H}_{66}$ \\
\hline 603 & 2415 & $H_{1}, H_{2}, \ldots . H_{69}$ \\
\hline 693 & 2775 & $\mathrm{H}_{1}, \mathrm{H}_{2}, \ldots . \mathrm{H}_{74}$ \\
\hline 750 & 3003 & $\mathrm{H}_{1}, \mathrm{H}_{2}, \ldots . . \mathrm{H}_{77}$ \\
\hline 850 & 3403 & $\mathrm{H}_{1}, \mathrm{H}_{2}, \ldots . \mathrm{H}_{82}$ \\
\hline 913 & 3655 & $\mathrm{H}_{1}, \mathrm{H}_{2}, \ldots . \mathrm{H}_{85}$ \\
\hline 1023 & 4095 & $\mathrm{H}_{1}, \mathrm{H}_{2}, \ldots \ldots \mathrm{H}_{90}$ \\
\hline 1092 & 4371 & $\mathrm{H}_{1}, \mathrm{H}_{2}, \ldots . \mathrm{H}_{93}$ \\
\hline 1212 & 4851 & $\mathrm{H}_{1}, \mathrm{H}_{2}, \ldots . \mathrm{H}_{98}$ \\
\hline 1287 & 5151 & $\mathrm{H}_{1}, \mathrm{H}_{2}, \ldots . \mathrm{H}_{101}$ \\
\hline
\end{tabular}


Proofs of the following three theorems follow the same arguments of Theorem 4.1.

Theorem 4.2 A complete tripartite graph $\mathrm{K}_{2,3, \mathrm{~m}}$ accepts $\mathrm{CMD}$ of $\mathrm{H}_{1}, \mathrm{H}_{2}, \ldots \ldots$, $H_{(5 n+7) / 2}$ if and only if $m=\left(5 n^{2}+16 n+3\right) / 8$ when $n$ is odd and $C M D$ of $H_{1}, H_{2}$, $\ldots . ., H_{(5 n+6) / 2}$ if and only if $m=\left(5 n^{2}+14 n\right) / 8$ when $n$ is even $\forall n \in \mathbb{N}$.

\section{Example 4.3}
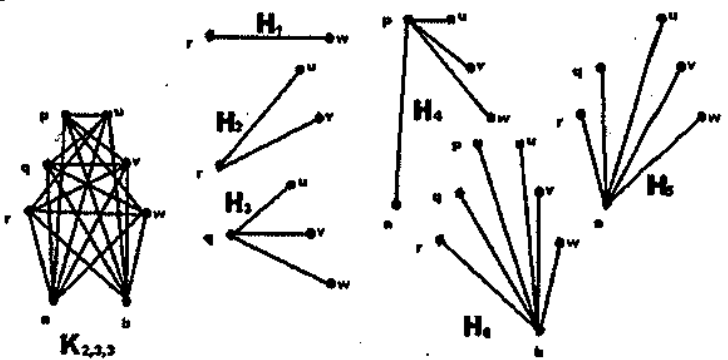

Fig 4.3: Continuous Monotonic Decomposition of $\mathrm{K}_{2,3,3}$

Table 4.2 First $25 \mathrm{~K}_{2,3, \mathrm{~m}}$ 's which admit $\mathrm{CMD}$ and their CMDs

\begin{tabular}{|c|c|c|}
\hline$m$ & $q\left(\mathrm{~K}_{2,3, \mathrm{~m}}\right)$ & \multicolumn{1}{c|}{$\mathrm{CMD}$} \\
\hline 3 & 21 & $\mathrm{H}_{1}, \mathrm{H}_{2}, \ldots . \mathrm{H}_{6}$ \\
\hline 6 & 36 & $\mathrm{H}_{1}, \mathrm{H}_{2}, \ldots . \mathrm{H}_{8}$ \\
\hline 12 & 66 & $\mathrm{H}_{1}, \mathrm{H}_{2}, \ldots . \mathrm{H}_{11}$ \\
\hline 17 & 91 & $\mathrm{H}_{1}, \mathrm{H}_{2}, \ldots . \mathrm{H}_{13}$ \\
\hline 26 & 136 & $\mathrm{H}_{1}, \mathrm{H}_{2}, \ldots . \mathrm{H}_{16}$ \\
\hline 33 & 171 & $\mathrm{H}_{1}, \mathrm{H}_{2}, \ldots . \mathrm{H}_{18}$ \\
\hline 45 & 231 & $\mathrm{H}_{1}, \mathrm{H}_{2}, \ldots . \mathrm{H}_{21}$ \\
\hline 54 & 276 & $\mathrm{H}_{1}, \mathrm{H}_{2}, \ldots . \mathrm{H}_{23}$ \\
\hline 69 & 351 & $\mathrm{H}_{1}, \mathrm{H}_{2}, \ldots . \mathrm{H}_{26}$ \\
\hline 80 & 406 & $\mathrm{H}_{1}, \mathrm{H}_{2}, \ldots . \mathrm{H}_{28}$ \\
\hline 98 & 496 & $\mathrm{H}_{1}, \mathrm{H}_{2}, \ldots . . \mathrm{H}_{31}$ \\
\hline 111 & 561 & $\mathrm{H}_{1}, \mathrm{H}_{2}, \ldots . \mathrm{H}_{33}$ \\
\hline
\end{tabular}

\begin{tabular}{|c|c|c|}
\hline$m$ & $q\left(\mathrm{~K}_{2,3, \mathrm{~m}}\right)$ & CMD \\
\hline 132 & 666 & $\mathrm{H}_{1}, \mathrm{H}_{2}, \ldots . \mathrm{H}_{36}$ \\
\hline 147 & 741 & $\mathrm{H}_{1}, \mathrm{H}_{2}, \ldots . \mathrm{H}_{38}$ \\
\hline 171 & 861 & $\mathrm{H}_{1}, \mathrm{H}_{2} \ldots \ldots \mathrm{H}_{41}$ \\
\hline 188 & 946 & $\mathrm{H}_{1}, \mathrm{H}_{2}, \ldots . \mathrm{H}_{43}$ \\
\hline 215 & 1081 & $\mathrm{H}_{1}, \mathrm{H}_{2}, \ldots . \mathrm{H}_{46}$ \\
\hline 234 & 1176 & $\mathrm{H}_{1}, \mathrm{H}_{2}, \ldots . \mathrm{H}_{48}$ \\
\hline 264 & 1326 & $\mathrm{H}_{1}, \mathrm{H}_{2}, \ldots . \mathrm{H}_{51}$ \\
\hline 285 & 1431 & $\mathrm{H}_{1}, \mathrm{H}_{2}, \ldots . \mathrm{H}_{53}$ \\
\hline 318 & 1596 & $\mathrm{H}_{1}, \mathrm{H}_{2}, \ldots . \mathrm{H}_{56}$ \\
\hline 341 & 1711 & $\mathrm{H}_{1}, \mathrm{H}_{2}, \ldots . \mathrm{H}_{58}$ \\
\hline 377 & 1891 & $\mathrm{H}_{1}, \mathrm{H}_{2}, \ldots . \mathrm{H}_{61}$ \\
\hline 402 & 2016 & $\mathrm{H}_{1}, \mathrm{H}_{2}, \ldots . \mathrm{H}_{63}$ \\
\hline 441 & 2211 & $\mathrm{H}_{1}, \mathrm{H}_{2}, \ldots . \mathrm{H}_{66}$ \\
\hline
\end{tabular}


Theorem 4.3 A complete tripartite graph $\mathrm{K}_{2,5, \mathrm{~m}}$ accepts $\mathrm{CMD}$ of $\mathrm{H}_{1}, \mathrm{H}_{2}, \ldots ., \mathrm{H}_{7 n+2}$ and $H_{1}, H_{2}, \ldots ., H_{7 n+4}$ if and only if $m=\left(7 n^{2}+5 n-2\right) / 2$ and $m=\left(7 n^{2}+9 n\right) / 2$ respectively, $\forall n \in \mathbb{N}$.

\section{Example 4.4}
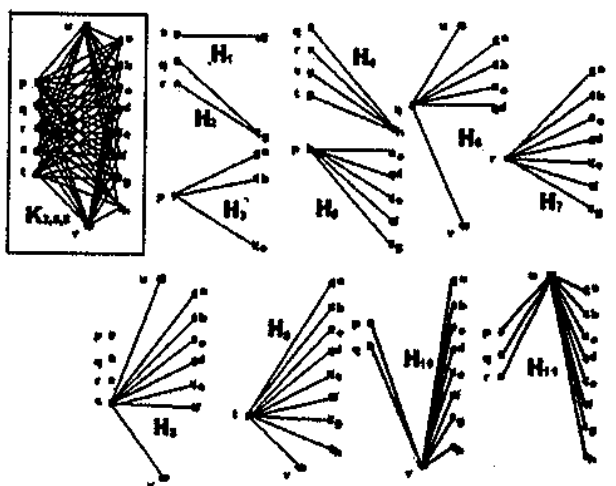

Fig 4.4: Continuous Monotonic Decomposition of $K_{2,5,8}$

Table 4.3 First $25 \mathrm{~K}_{2,5, \mathrm{~m}}$ 's which admit CMD and their CMDs

\begin{tabular}{|c|c|c|}
\hline$m$ & $q\left(K_{2,3, m}\right)$ & $C M D$ \\
\hline 5 & 45 & $H_{1}, H_{2}, \ldots . H_{9}$ \\
\hline 8 & 66 & $H_{1}, H_{2}, \ldots . H_{11}$ \\
\hline 18 & 136 & $H_{1}, H_{2}, \ldots . . H_{16}$ \\
\hline 23 & 171 & $H_{1}, H_{2}, \ldots . . H_{18}$ \\
\hline 38 & 276 & $H_{1}, H_{2}, \ldots \ldots H_{23}$ \\
\hline 45 & 325 & $H_{1}, H_{2}, \ldots . . H_{25}$ \\
\hline 65 & 465 & $H_{1}, H_{2}, \ldots . H_{30}$ \\
\hline 74 & 528 & $H_{1}, H_{2}, \ldots . . H_{32}$ \\
\hline 99 & 703 & $H_{1}, H_{2}, \ldots \ldots H_{37}$ \\
\hline 110 & 780 & $H_{1}, H_{2}, \ldots . . H_{39}$ \\
\hline 140 & 990 & $H_{1}, H_{2}, \ldots \ldots H_{44}$ \\
\hline 153 & 1081 & $H_{1}, H_{2}, \ldots . . H_{46}$ \\
\hline
\end{tabular}

\begin{tabular}{|c|c|c|}
\hline $\mathrm{m}$ & $\mathrm{q}\left(\mathrm{K}_{2,3, \mathrm{~m}}\right)$ & CMD \\
\hline 188 & 1326 & $\mathrm{H}_{1}, \mathrm{H}_{2}, \ldots . \mathrm{H}_{51}$ \\
\hline 203 & 1431 & $H_{1}, H_{2}, \ldots, H_{53}$ \\
\hline 243 & 1711 & $H_{1}, H_{2}, \ldots . . H_{58}$ \\
\hline 260 & 1830 & $\mathrm{H}_{1}, \mathrm{H}_{2}, \ldots . \mathrm{H}_{60}$ \\
\hline 305 & 2145 & $\mathrm{H}_{1}, \mathrm{H}_{2}, \ldots \ldots \mathrm{H}_{65}$ \\
\hline 324 & 2278 & $H_{1}, H_{2}, \ldots . . H_{67}$ \\
\hline 374 & 2628 & $\mathrm{H}_{1}, \mathrm{H}_{2}, \ldots \ldots \mathrm{H}_{72}$ \\
\hline 395 & 2775 & $H_{1}, H_{2}, \ldots . H_{74}$ \\
\hline 450 & 3160 & $\mathrm{H}_{1}, \mathrm{H}_{2}, \ldots . \mathrm{H}_{79}$ \\
\hline 473 & 3321 & $\mathrm{H}_{1}, \mathrm{H}_{2}, \ldots . \mathrm{H}_{81}$ \\
\hline 533 & 3741 & $H_{1}, H_{2}, \ldots . H_{86}$ \\
\hline 558 & 3916 & $H_{1}, H_{2}, \ldots . H_{88}$ \\
\hline 623 & 4371 & $H_{1}, H_{2}, \ldots . . H_{93}$ \\
\hline
\end{tabular}


Theorem 4.4 A complete tripartite graph $K_{3,5, \mathrm{~m}}$ accepts $C M D$ of $H_{1}, H_{2}, \ldots$. , $H_{16 n .6}$ and $H_{1}, H_{2}, \ldots ., H_{16 n+5}$ if and only if $m=16 n^{2}-11 n$ and $m=16 n^{2}+11 n$ respectively, $\forall n \in \mathbb{N}$.

\section{Example 4.5}

Let us consider the graph $\mathrm{K}_{3,5,5}$.

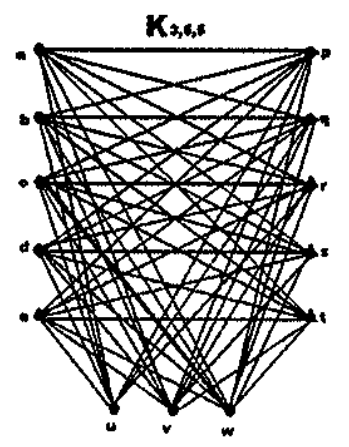

Fig 4.5: $\mathrm{K}_{3,5,5}$

Let the three sets of vertices be $V_{1}=\{u, v, w\} \quad V_{2}=\{a, b, c, d, e\}$ and $V_{3}=\{p, q, r, s, t\}$.

Continuous Monotonic Decomposition of $\mathrm{K}_{3,5,5}$ is as follows:

$$
\begin{aligned}
& H_{1}=\{(q, e)\} \\
& H_{2}=\{(p, a),(p, b)\} \\
& H_{3}=\{(p, c),(p, d),(p, e)\} \\
& H_{4}=\{(q, a),(q, b),(q, c),(q, d)\} \\
& H_{s}=\{(r, a),(r, b),(r, c),(r, d),(r, e),\} \\
& H_{6}=\{(s, a),(s, b),(s, c),(s, d),(s, e),(s, w)\} \\
& H_{7}=\{(t, a),(t, b),(t, c),(t, d),(t, e),(t, v),(t, w)\} \\
& H_{8}=\{(w, a),(w, b),((w, c),(w, d),(w, e),(w, p),(w, q),(w, r)\} \\
& H_{9}=\{(v, a),(v, b),(v, c),(v, d),(v, e),(v, p),(v, q),(v, r),(v, s),\} \\
& H_{10}=\{(u, a),(u, b),(u, c),(u, d),(u, e),(u, p),(u, q),(u, r),(u, s),(u, t)\}
\end{aligned}
$$


Table 4.4 First $25 \mathrm{~K}_{3,5, \mathrm{~m}}$ 's which admit CMD and their CMDs

\begin{tabular}{|c|c|c|}
\hline$m$ & $q\left(\mathrm{~K}_{2,3, \mathrm{~m}}\right)$ & $\mathrm{CMD}$ \\
\hline 5 & 55 & $\mathrm{H}_{1}, \mathrm{H}_{2}, \ldots . \mathrm{H}_{10}$ \\
\hline 27 & 231 & $\mathrm{H}_{1}, \mathrm{H}_{2}, \ldots . \mathrm{H}_{21}$ \\
\hline 42 & 351 & $\mathrm{H}_{1}, \mathrm{H}_{2}, \ldots . \mathrm{H}_{26}$ \\
\hline 86 & 703 & $\mathrm{H}_{1}, \mathrm{H}_{2} \ldots \ldots \mathrm{H}_{37}$ \\
\hline 111 & 903 & $\mathrm{H}_{1}, \mathrm{H}_{2} \ldots \ldots \mathrm{H}_{42}$ \\
\hline 177 & 1431 & $\mathrm{H}_{1}, \mathrm{H}_{2}, \ldots . \mathrm{H}_{53}$ \\
\hline 212 & 1711 & $\mathrm{H}_{1}, \mathrm{H}_{2}, \ldots \ldots \mathrm{H}_{58}$ \\
\hline 300 & 2415 & $\mathrm{H}_{1}, \mathrm{H}_{2}, \ldots . \mathrm{H}_{69}$ \\
\hline 345 & 2775 & $\mathrm{H}_{1}, \mathrm{H}_{2}, \ldots . \mathrm{H}_{74}$ \\
\hline 455 & 3655 & $\mathrm{H}_{1}, \mathrm{H}_{2}, \ldots . \mathrm{H}_{85}$ \\
\hline 510 & 4095 & $\mathrm{H}_{1}, \mathrm{H}_{2}, \ldots . \mathrm{H}_{90}$ \\
\hline 642 & 5151 & $\mathrm{H}_{1}, \mathrm{H}_{2}, \ldots . \mathrm{H}_{101}$ \\
\hline
\end{tabular}

\begin{tabular}{|c|c|c|}
\hline$m$ & $q\left(\mathrm{~K}_{2,3, \mathrm{~m}}\right)$ & $C M D$ \\
\hline 707 & 5671 & $\mathrm{H}_{1}, \mathrm{H}_{2} \ldots \ldots \mathrm{H}_{106}$ \\
\hline 861 & 6903 & $\mathrm{H}_{1}, \mathrm{H}_{2}, \ldots . . \mathrm{H}_{117}$ \\
\hline 936 & 7503 & $\mathrm{H}_{1}, \mathrm{H}_{2}, \ldots . \mathrm{H}_{122}$ \\
\hline 1112 & 8911 & $\mathrm{H}_{1}, \mathrm{H}_{2}, \ldots . \mathrm{H}_{133}$ \\
\hline 1197 & 9591 & $\mathrm{H}_{1}, \mathrm{H}_{2}, \ldots . \mathrm{H}_{138}$ \\
\hline 1395 & 11175 & $\mathrm{H}_{1}, \mathrm{H}_{2}, \ldots . \mathrm{H}_{149}$ \\
\hline 1490 & 11935 & $\mathrm{H}_{1}, \mathrm{H}_{2}, \ldots . \mathrm{H}_{154}$ \\
\hline 1710 & 13695 & $\mathrm{H}_{1}, \mathrm{H}_{2}, \ldots . \mathrm{H}_{185}$ \\
\hline 1815 & 14535 & $\mathrm{H}_{1}, \mathrm{H}_{2}, \ldots . \mathrm{H}_{170}$ \\
\hline 2057 & 16471 & $\mathrm{H}_{1}, \mathrm{H}_{2}, \ldots . \mathrm{H}_{181}$ \\
\hline 2172 & 17391 & $\mathrm{H}_{1}, \mathrm{H}_{2}, \ldots . . \mathrm{H}_{186}$ \\
\hline 2436 & 19503 & $\mathrm{H}_{1}, \mathrm{H}_{2}, \ldots . \mathrm{H}_{197}$ \\
\hline 2561 & 20503 & $\mathrm{H}_{1}, \mathrm{H}_{2}, \ldots . . \mathrm{H}_{202}$ \\
\hline
\end{tabular}

\section{Conclusion}

The results described above are about four complete tripartite graphs that accept $C M D$. There are many other classes of complete tripartite graphs that accept CMD. Study can be extended to find the algorithms for the above graphs to accept Continuous Monotonic Star Decomposition (CMSD) and Continuous Monotonic Path Decomposition (CMPD). Finding the size of the graph is the major task in the process. The study can also be extended to complete $m$-partite graphs for greater values of $m$.

\section{References}

[1] F. Harary, Groph Theory, Addison-Wesley Publishing House, USA, 1969.

[2] Y. Alavi, A. J. Boais, G. Chartrand, P. Eros and O.R. Ollermann, "The Ascending Subgraph Decomposition Problem," Congressus Numerantium, 1987, Vol. 58, p.7-14 
[3] N. Gnana Dhas and J. Poulraj Joseph, "Continuous Monotonic Decomposition of Graphs," International Journal of Management and Systems, Vol 16, No. 3, Sept-Dec, 2000, pp. 333-344

[4] N. Gnana Dhas and J. Paulraj Joseph, "Continuous Monotonic Decomposition of Cycles," International Journal of Management and Systems, Vol 19, No. 1, Jan-April, 2003, pp. 65 76.

[5] A Nagarajan and S. Navaneetha Krishnan, "Continuous Monotonic Decomposition of Some Special Class of Graphs," International Journal of Management and Systems, Vol. 21, No.1, Jan-Apr, 2005, pp. 91-106.

[6] D. M. Burton, Elementary Number Theory, New Delhi: Universal Book Stall, 1998. 